\title{
Editorial: A Compendium of Recent Research on Stem Cell-Based Therapy for Covid-19
}

\begin{abstract}
Abdelkrim Hmadcha ${ }^{1,2,3 *}$, Bernat Soria ${ }^{2,4}$, Robert C. Zhao ${ }^{5,6,7}$, Tarik Smani ${ }^{8,9}$ and Israel Valverde ${ }^{10,11}$

${ }^{1}$ Department of Biotechnology, University of Alicante, Alicante, Spain, ${ }^{2}$ University of Pablo de Olavide, Seville, Spain, ${ }^{3}$ Spanish Biomedical Research Centre in Diabetes and Associated Metabolic Disorders (CIBERDEM), Madrid, Spain, ${ }^{4}$ Department of Physiology, Institute of Bioengineering-ISABIAL, University Miguel Hernández School of Medicine, Alicante, Spain, ${ }^{5}$ Institute of Basic Medical Sciences, Chinese Academy of Medical Sciences, School of Basic Medicine, Peking Union Medical College, Beijing, China, ${ }^{6}$ School of Life Sciences, Shanghai University, Shanghai, China, ${ }^{7}$ International Society on Aging and Disease, Bryan, TX, United States, ${ }^{8}$ Cardiovascular Pathophysiology, Institute of Biomedicine of Seville, University Hospital of Virgen del Rocio, University of Seville, CSIC, Seville, Spain, ${ }^{9}$ Department of Medical Physiology and Biophysics, University of Seville, Seville, Spain, ${ }^{10}$ Pediatric Cardiology Unit, Virgen del Rocio University Hospital, Seville, Spain, ${ }^{11}$ Institute of Biomedicine of Seville, University Hospital of Virgen del Rocío, University of Seville, CSIC, Seville, Spain
\end{abstract}

Keywords: advanced therapies, clinical trials, cell therapy, mesenchymal stromal cells, CAR T cells, COVID-19, cytokine storm, immunomodulaion

\section{Editorial on the Research Topic}

A Compendium of Recent Research on Stem Cell-Based Therapy for Covid-19

\section{OPEN ACCESS}

Edited and reviewed by: Valerie Kouskoff,

The University of Manchester, United Kingdom

${ }^{*}$ Correspondence: Abdelkrim Hmadcha khmadcha@upo.es

Specialty section: This article was submitted to

Stem Cell Research,

a section of the journal Frontiers in Cell and Developmental

Biology

Received: 11 November 2021 Accepted: 18 November 2021

Published: 14 December 2021

Citation:

Hmadcha A, Soria B, Zhao RC Smani T and Valverde I (2021) Editorial: A Compendium of Recent Research on Stem Cell-Based Therapy for Covid-

Front. Cell Dev. Biol. 9:813384. doi: 10.3389/fcell.2021.813384

\section{INTRODUCTION}

We have been dealing with an unprecedented global health crisis for nearly 2 years now. This started after an outbreak of atypical pneumonia of unknown etiology that was described in late December 2019 in China's Wuhan Province, the etiologic agent causing this pneumonia episode was identified as a novel coronavirus named "Severe Acute Respiratory Syndrome CoronaVirus-2" (SARS-CoV-2)" (Coronaviridae Study Group of the International Committee on Taxonomy of Viruses, 2020; Wu et al., 2020) and the disease was designated COronaVIrus Disease-2019 (COVID-19). The rapid expansion of COVID-19 cases in number and geographic distribution led the World Health Organization (WHO) to declare a global health emergency. The control of the disease was challenged by the lack of antiviral treatment and vaccines, by asymptomatic carriers and the rapid increase in infections worldwide; COVID-19 was officially classified and declared by WHO as a pandemic on March 11, 2020 (WHO, 2020).

COVID-19 affects people differently, the majority of infected individuals develop mild to moderate disease and recover without hospitalization, but a subgroup of patients progresses to severe disease, with a high mortality rate and limited treatment options. However, the clinical features of COVID-19 vary from asymptomatic forms to conditions involving multi-organ and systemic manifestations in terms of septic shock and multiple organ dysfunction syndrome (MODS). Common primary pathologic features of critical COVID-19 overlap with acute lung injury (ALI) and acute respiratory distress syndrome (ARDS). While most infected individuals are usually asymptomatic or have mild symptoms, about $15 \%$ are affected by ARDS, of which $5 \%$ progress to multiple organ dysfunction syndrome or failure (Hui and Zumla, 2019).

COVID-19 involves direct attacks by SARS-CoV-2 on cells and secondary attacks on the body following activation of the immune system. Consequently, both the virus and the immune response 
can cause damage to the body, leading to common complications or secondary infection. The spike protein (S-protein) of SARS$\mathrm{CoV}-2$ binds to its functional receptor angiotensin-converting enzyme 2 (ACE2), triggering endocytosis of virus particles to infect target cells (Li et al., 2003; Tay et al., 2020). Therefore, potential therapeutic candidates for studying the mechanisms of SARS-CoV-2 infection include the ACE2 receptor (Zhang et al., 2020).

Given that the exact pathogenesis of SARS-CoV-2 and the dynamics of the disease are not yet fully understood, nor are specific antiviral options available, makes the treatment of patients with COVID-19 still a challenge (Tay et al., 2020); therefore, the available treatment options are limited. Several antiviral drugs (Grein et al., 2020), corticosteroids (Wang et al., 2020; Rasheedi et al., 2021), convalescent plasma (Shen et al., 2020; Verma et al., 2020) and neutralizing monoclonal antibodies (Shanmugaraj et al., 2020) have been tested and have undergone different phases of clinical trials, but none have been approved for COVID-19. Another alarming development is the recurrence of infection in recovered and even vaccinated individuals, which challenges the efficacy of current treatments (Lan et al., 2020). Under this situation, research has been carried out at an unprecedented speed to achieve a vaccine. Although several vaccines are now available and millions of people have received their full dose, the process of assessing safety, efficacy is still challenged, and a longer follow-up is still required (Kadkhoda, 2021; Olliaro et al., 2021; Yan et al., 2021). While the efficacy of vaccines has focused on the reduction of the number of symptomatic cases, the durability of acquired immunity following vaccination, the protection against reinfection by SARS-CoV-2 and its emerging variants remain to be proven and even improved (Townsend et al., 2021).

Thus, there is an urgent need for the development of feasible, safe and effective therapies. Therefore, growing experimental and clinical evidence suggests that advanced therapies could provide a potential therapeutic alternative for COVID-19 caused by SARSCoV-2 and its emerging variants. In particular, cell therapy has been proven to significantly improve the sequelae related to COVID-19. In this regard, MSCs are among the most frequently used cell type for cell therapy, and considerable efforts have been made to introducing this advanced cell-based therapy into clinical practice. MSCs have been established as promising candidate sources for cell-based therapy their broad pharmacological effects and due to their contributions to tissue and organ homeostasis, repair and support by self-renewal and multi-differentiation, as well as by their anti-inflammatory, antiproliferative, immunomodulatory, pro-angiogenic, pleiotropic, tropic and trophic properties. Various diseases have been successfully treated by MSCs in animal models and hundreds of clinical trials related to the potential benefits of MSCs are ongoing or have been successfully concluded so far. MSCs also secrete a myriad of soluble factors and vesicles altogether contribute to tissues and organs support, repair, homeostasis and functionality. The efficacy of MSCs and their secretory factors has been proven in successfully reducing inflammation, dampening immune responses and repairing lung damage in various pre-clinical and clinical models (Hmadcha et al., 2009).
As described in the review by Ligotti et al., the key roles of both immunosenescence and immunopathology in the outcome of SARS-CoV-2 infection are supported by the beneficial results obtained with infusion of MSCs that act by restoring immune homeostasis and contributing to lung repair. In addition, the potential of MSC-based therapy as an option for severe or critically ill COVID-19 patients has been explored (Leng et al., 2020; Sánchez-Guijo et al., 2020). Several studies focus on regenerative, immunomodulatory, and anti-inflammatory properties of mesenchymal stromal cells (MSCs) to reduce the manifestation of cytokine storm and to restore ARDS and ALI, exhibiting an important option to be applied to critical COVID19 patients; or on MSCs secretome to treat COVID-19 pneumonia (Li et al., 2020; Liang et al., 2020; Meng et al., 2020; Tang et al., 2020; Lanzoni et al., 2021). The contribution of Arjmand et al., summarizes the research on cytokine storm, one of the key causes of MODS as a hallmark of COVID-19 severity, highlights the benefit of stem cell-based therapies to attenuate cytokine release syndrome and suggests emerging advantages of MSCs secretome and extracellular vesicles (EVs) as treatment approaches for COVID-19. Besides, 2 interesting hypotheses and theories are formulated on the one hand, Nazerian et al., hypothesize that the use of chimeric 8P9R peptide and soluble ACE2 using exosome-liposome hybrids in the form of a two-step phase-dependent therapeutic strategy could inhibit the viral intracellular pathway and also inhibit progression in cytokine storm in a personalized manner. Given that this strategy is sensitive to the inflammatory status of individuals, it may improve the outcome of COVID-19 mortality. On the other hand, Babajani et al., provided new shreds of evidence about mitochondrial dysfunction and its effects on the immune response in COVID-19 and hypothesized that in vivo and in vitro experimental transferring healthy mitochondria by MSCs to damaged cells can provide a new therapeutic approach for COVID-19.

Within the cell therapy approach, other research includes the use of hematopoietic stem cells derived from umbilical cord blood, bone marrow, or mobilized peripheral blood, as well as immune chimeric antigen receptor $\mathrm{T}$-cell (CAR-T cells) (Vardhana and Wolchok, 2020). Of note, an interesting immunology and virology original paper by Li et al., provided data that can further improve development of vaccines and new therapies against COVID-19. In this regard, authors reported a series of potential epitopes on SARS-CoV-2 spike glycoprotein which are recognized by $\mathrm{CD}^{+}$and $\mathrm{CD}^{+} \mathrm{T}$ cells from patients recovered from COVID-19 in China. The authors found that $\mathrm{CD}_{134^{+}}$and $\mathrm{CD} 137^{+} \mathrm{T}$ cells can react to an epitope outside the spike receptor domain (RBD), isolated T-cell receptor (TCR) sequences from the immunoreactive $\mathrm{T}$ cells and also inserted into Jurkat and $\mathrm{CD}^{+} \mathrm{T}$ cells to identify an epitope-specific TCR. Ferreras et al., for their part, characterized SARS-CoV-2-specific $\mathrm{T}$-cell population within the CD45RA ${ }^{-}$memory $\mathrm{T}$ cells (either $\mathrm{CD}^{+}$or $\mathrm{CD}^{+}$) from blood of convalescent donors. In addition, the authors have demonstrated that these cells can be easily, effectively, and rapidly isolated following a donor selection strategy based on IFN- $\gamma$ expression after exposure with SARSCoV-2-specific peptides and HLA antigen expression, thereby 
obtaining clinical-grade CD45RA ${ }^{-}$memory $\mathrm{T}$ cells, without requirements for GMP conditions, allowing the establishment of a biobank of SARS-CoV-2 specific memory T cells to be used to treat moderate to severe cases of COVID-19 patients.

The understanding of the mechanism of infection and pathogenesis also have great appeal and are still limited. In this regard; the use of human pluripotent stem cells, both embryonic stem cells (hESCs) and induced stem cells (hiPSCs), to generate tissue-specific human organoids (lung, intestinal, liver, vascular, heart, and kidney organoids) may provide a next-generation cellular model for investigating viral infection and drug screening (Yang et al., 2020). In this context, Larijani et al., reviewed the currently available iPSC-derived cells, iPSC-derived organoid models and animal models for studying the pathophysiology mechanisms of COVID-19-associated disorders and discussed the challenges and limitations that need to be overcome to optimize modelling approaches for the disease, and proposed potential therapeutic advances identified in experimental studies. Relatedly, in their review Luo et al., recapitulated findings on the application of hiPSC-derived cell models and organoids for COVID-19 to understand the action of SARS-COV-2 on human cells, underlining the importance of developing these models for longterm experiments not only to study the pathology of SARS-CoV-2 infection, respiratory failure and dysregulation of organs and systems, but also, to mimic the natural host-virus interaction, to clarify viral infection mechanisms and to elucidate the in vivo conditions of viral life cycles and drug screening.

We would like to conclude referring to the opinion by Zhao CR and others, pioneer in using MSCs to improve the

\section{REFERENCES}

Coronaviridae Study Group of the International Committee on Taxonomy of Viruses (2020). The Species Severe Acute Respiratory Syndrome-Related Coronavirus: Classifying 2019-nCoV and Naming it SARS-CoV-2. Nat. Microbiol. 5 (4), 536-544. eng. Epub 20200302. doi:10.1038/s41564-0200695-z

Grein, J., Ohmagari, N., Shin, D., Diaz, G., Asperges, E., Castagna, A., et al. (2020). Compassionate Use of Remdesivir for Patients with Severe Covid-19. N. Engl. J. Med. 382 (24), 2327-2336. eng. Epub 20200410. doi:10.1056/ NEJMoa2007016

Hmadcha, A., Abdelkrim, H., Domínguez-Bendala, J., Juan, D. B., Wakeman, J., Jane, W., et al. (2009). The Immune Boundaries for Stem Cell Based Therapies: Problems and Prospective Solutions. J. Cel Mol Med 13 (8A), 1464-1475. eng. Epub 20090706. doi:10.1111/j.1582-4934.2009.00837.x

Hui, D. S. C., and Zumla, A. (2019). Severe Acute Respiratory Syndrome. Infect. Dis. Clin. North America 33 (4), 869-889. Cited in: Pubmed; PMID 31668196. doi:10.1016/j.idc.2019.07.001

Kadkhoda, K. (2021). Post-adenoviral-based COVID-19 Vaccines Thrombosis: A Proposed Mechanism. J. Thromb. Haemost. 19 (7), 1831-1832. eng. Epub 20210505. doi: $10.1111 /$ jth. 15348

Lan, L., Xu, D., Ye, G., Xia, C., Wang, S., Li, Y., et al. (2020). Positive RT-PCR Test Results in Patients Recovered from COVID-19. JAMA 323 (15), 1502-1503. eng. doi:10.1001/jama.2020.2783

Lanzoni, G., Linetsky, E., Correa, D., Messinger Cayetano, S., Alvarez, R. A., Kouroupis, D., et al. (2021). Umbilical Cord Mesenchymal Stem Cells for COVID -19 Acute Respiratory Distress Syndrome: A Double-blind, Phase 1/2a, Randomized Controlled Trial. Stem Cell Transl Med 10 (5), 660-673. eng. Epub 20210105. doi:10.1002/sctm.20-0472 outcome in patients with COVID-19 pneumonia (Leng et al., 2020). Wang et al., bring the debate about the clinical use of MSCs and MSCs-derived EVs for combating COVID-19, the risk of their uncontrolled commercial application to the forefront and the lack of any suggestions on regulations and guidelines for regulatory agencies to adopt new policies to prevent the sale of unproven MSC-based treatments in COVID patients.

Here we assembled a compendium of 9 manuscripts (2 original research, 4 reviews, 2 hypothesis and theory and 1 opinion) that cover the scope of this research topic, thus highlighting some of the recent advances and progresses of preclinical and clinical research and discussing some of the critical aspects related to the application of stem cell for COVID-19. Altogether, the ultimate goal of all these strategies is to achieve a safe and controlled therapy for COVID-19.

\section{AUTHOR CONTRIBUTIONS}

All authors listed have made a substantial, direct, and intellectual contribution to the work and approved it for publication.

\section{ACKNOWLEDGMENTS}

We thank all contributing authors, reviewers and Frontiers journal editorial team for their support to this Research Topic.

Leng, Z., Zhu, R., Hou, W., Feng, Y., Yang, Y., Han, Q., et al. (2020). Transplantation of ACE2- Mesenchymal Stem Cells Improves the Outcome of Patients with COVID-19 Pneumonia. Aging Dis. 11 (2), 216-228. doi:10.14336/AD.2020.0228

Li, W., Moore, M. J., Vasilieva, N., Sui, J., Wong, S. K., Berne, M. A., et al. (2003). Angiotensin-converting Enzyme 2 Is a Functional Receptor for the SARS Coronavirus. Nature 426 (6965), 450-454. Pubmed; PMID 14647384. doi: $10.1038 /$ nature 02145

Li, Z., Niu, S., Guo, B., Gao, T., Wang, L., Wang, Y., et al. (2020). Stem Cell Therapy for COVID-19, ARDS and Pulmonary Fibrosis. Cell Prolif 53 (12), e12939, 2020 eng. Epub 20201024. doi:10.1111/cpr.12939

Liang, B., Chen, J., Li, T., Wu, H., Yang, W., Li, Y., et al. (2020). Clinical Remission of a Critically Ill COVID-19 Patient Treated by Human Umbilical Cord Mesenchymal Stem Cells. Medicine (Baltimore) 99 (31), e21429. doi:10.1097/MD.0000000000021429

Meng, F., Xu, R., Wang, S., Xu, Z., Zhang, C., Li, Y., et al. (2020). Human Umbilical Cord-Derived Mesenchymal Stem Cell Therapy in Patients with COVID-19: a Phase 1 Clinical Trial. Sig Transduct Target. Ther. 5 (1), 172. doi:10.1038/ s41392-020-00286-5

Olliaro, P., Torreele, E., and Vaillant, M. (2021). COVID-19 Vaccine Efficacy and Effectiveness-The Elephant (Not) in the Room. Lancet Microbe 2 (7), e279-e280. eng. We declare no competing interests. Epub 20210420. doi:10.1016/S2666-5247(21)00069-0

Rasheedi, M. A., Alhazmi, Y., Almaqwashi, N., Mateq Ali, A., Kardam, A., Sharaf, M., et al. (2021). Corticosteroid Therapy for 2019-nCoV-infected Patients: A Case Series of Eight Mechanically Ventilated Patients. Clin. Case Rep. 9 (5), e04066, 2021 . eng. None declared. Epub 20210326. doi:10.1002/ccr3.4066

Sánchez-Guijo, F., García-Arranz, M., López-Parra, M., Monedero, P., MataMartínez, C., Santos, A., et al. (2020). Adipose-derived Mesenchymal Stromal Cells for the Treatment of Patients with Severe SARS-CoV-2 
Pneumonia Requiring Mechanical Ventilation. A Proof of Concept Study. EClinicalMedicine 25, 100454. doi:10.1016/j.eclinm.2020.100454

Shanmugaraj, B., Siriwattananon, K., Wangkanont, K., and Phoolcharoen, W. (2020). Perspectives on Monoclonal Antibody Therapy as Potential Therapeutic Intervention for Coronavirus Disease-19 (COVID-19). Asian Pac. J. Allergy Immunol. 38 (1), 10-18. doi:10.12932/AP-200220-0773

Shen, C., Wang, Z., Zhao, F., Yang, Y., Li, J., Yuan, J., et al. (2020). Treatment of 5 Critically Ill Patients with COVID-19 with Convalescent Plasma. JAMA 323 (16), 1582-1589. Pubmed; PMID 32219428. doi:10.1001/jama.2020.4783

Tang, L., Jiang, Y., Zhu, M., Chen, L., Zhou, X., Zhou, C., et al. (2020). Clinical Study Using Mesenchymal Stem Cells for the Treatment of Patients with Severe COVID-19. Front. Med. 14 (5), 664-673. eng. Epub 20200806. doi:10.1007/ s11684-020-0810-9

Tay, M. Z., Poh, C. M., Rénia, L., MacAry, P. A., and Ng, L. F. P. (2020). The trinity of COVID-19: Immunity, Inflammation and Intervention. Nat. Rev. Immunol. 20 (6), 363-374. eng. Epub 20200428. doi:10.1038/s41577-020-0311-8

Townsend, J. P., Hassler, H. B., Wang, Z., Miura, S., Singh, J., Kumar, S., et al. (2021). The Durability of Immunity against Reinfection by SARS-CoV-2: a Comparative Evolutionary Study. Lancet Microbe. doi:10.1016/S2666-5247(21) 00219-6

Vardhana, S. A., and Wolchok, J. D. (2020). The many Faces of the Anti-COVID Immune Response. J. Exp. Med. 217 (6), 678. doi:10.1084/jem.20200678

Verma, H. K., Farran, B., and Bhaskar, L. V. K. S. (2020). Convalescent Plasma Transfusion a Promising Therapy for Coronavirus Diseases 2019 (COVID-19): Current Updates. Antib Ther. 3 (2), 115-125. eng. Epub 20200527. doi:10.1093/ abt/tbaa010

Wang, Y., Jiang, W., He, Q., Wang, C., Liu, B., Zhou, P., et al. (2020). Early, LowDose and Short-Term Application of Corticosteroid Treatment in Patients with Severe COVID-19 Pneumonia: Single-center Experience from Wuhan. China. Medrxiv 2020, 32342. doi:10.1101/2020.03.06.20032342

WHO (2020). Novel Coronavirus (2019-nCoV). situation report, 22. Geneva, Switzerland: WHO.
Wu, F., Zhao, S., Yu, B., Chen, Y.-M., Wang, W., Song, Z.-G., et al. (2020). A New Coronavirus Associated with Human Respiratory Disease in China. Nature 579 (7798), 265-269. eng. Epub 20200203. doi:10.1038/s41586-020-2008-3

Yan, Y., Pang, Y., Lyu, Z., Wang, R., Wu, X., You, C., et al. (2021). The COVID-19 Vaccines: Recent Development, Challenges and Prospects. Vaccines (Basel) 9 (4), 349. doi:10.3390/vaccines 9040349

Yang, L., Han, Y., Nilsson-Payant, B. E., Gupta, V., Wang, P., Duan, X., et al. (2020). A Human Pluripotent Stem Cell-Based Platform to Study SARS-CoV-2 Tropism and Model Virus Infection in Human Cells and Organoids. Cell Stem Cell 27 (1), 125-136. doi:10.1016/j.stem.2020.06.015

Zhang, H., Penninger, J. M., Li, Y., Zhong, N., and Slutsky, A. S. (2020). Angiotensin-converting Enzyme 2 (ACE2) as a SARS-CoV-2 Receptor: Molecular Mechanisms and Potential Therapeutic Target. Intensive Care Med. 46 (4), 586-590. eng. Epub 20200303. doi:10.1007/s00134-020-05985-9

Conflict of Interest: The authors declare that the research was conducted in the absence of any commercial or financial relationships that could be construed as a potential conflict of interest.

Publisher's Note: All claims expressed in this article are solely those of the authors and do not necessarily represent those of their affiliated organizations, or those of the publisher, the editors and the reviewers. Any product that may be evaluated in this article, or claim that may be made by its manufacturer, is not guaranteed or endorsed by the publisher.

Copyright (๑) 2021 Hmadcha, Soria, Zhao, Smani and Valverde. This is an openaccess article distributed under the terms of the Creative Commons Attribution License (CC BY). The use, distribution or reproduction in other forums is permitted, provided the original author $(s)$ and the copyright owner(s) are credited and that the original publication in this journal is cited, in accordance with accepted academic practice. No use, distribution or reproduction is permitted which does not comply with these terms. 